\title{
Equation of state of Mo from shock compression experiments on preheated samples
}

O. V. Fat'yanov and P. D. Asimow

Citation: Journal of Applied Physics 121, 115904 (2017); doi: 10.1063/1.4978607

View online: http://dx.doi.org/10.1063/1.4978607

View Table of Contents: http://aip.scitation.org/toc/jap/121/11

Published by the American Institute of Physics

\section{Articles you may be interested in}

Loading-path dependent deformation of nanocrystalline Ta under single- and double-shock, and quasi-isentropic compression

Journal of Applied Physics 121, 115901115901 (2017); 10.1063/1.4978359

Refractive index and phase transformation of sapphire under shock pressures up to $210 \mathrm{GPa}$

Journal of Applied Physics 121, 115903115903 (2017); 10.1063/1.4978746

Acoustic Wood anomaly in transmitted diffraction field

Journal of Applied Physics 121, 114902114902 (2017); 10.1063/1.4978419

Elastic-plastic deformation of molybdenum single crystals shocked along [100]

Journal of Applied Physics 121, 045903045903 (2017); 10.1063/1.4974475

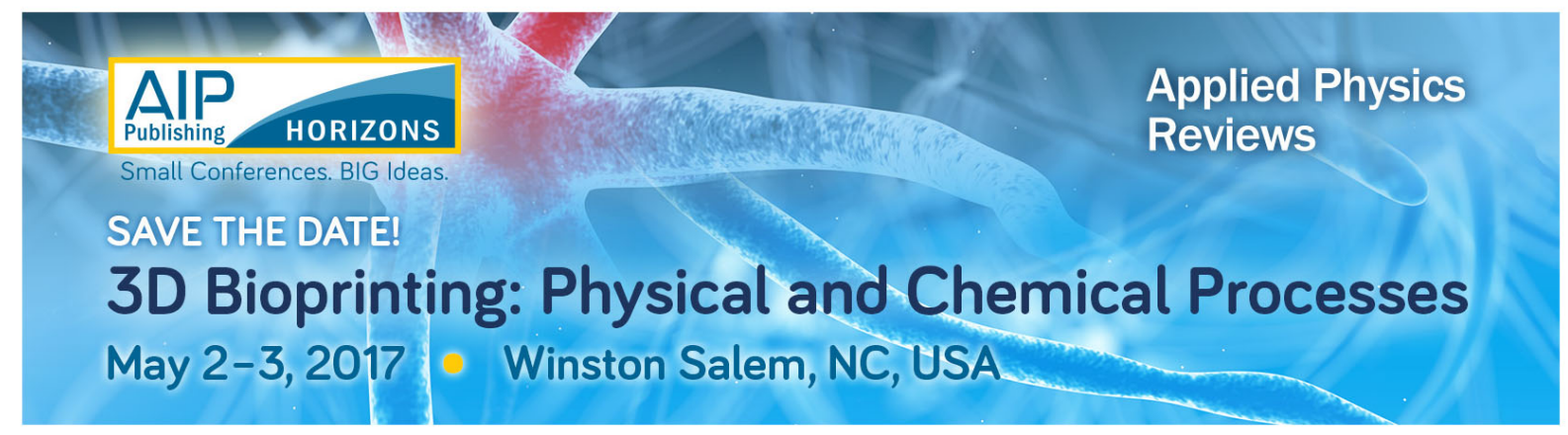




\title{
Equation of state of Mo from shock compression experiments on preheated samples
}

\author{
O. V. Fat'yanov ${ }^{\text {a) }}$ and P. D. Asimow ${ }^{\text {b) }}$ \\ Division of Geological and Planetary Sciences 252-21, California Institute of Technology, Pasadena, \\ California 91125, USA
}

(Received 22 November 2016; accepted 2 March 2017; published online 21 March 2017)

\begin{abstract}
We present a reanalysis of reported Hugoniot data for Mo, including both experiments shocked from ambient temperature $(T)$ and those preheated to $1673 \mathrm{~K}$, using the most general methods of least-squares fitting to constrain the Grüneisen model. This updated Mie-Grüneisen equation of state (EOS) is used to construct a family of maximum likelihood Hugoniots of Mo from initial temperatures of 298 to $2350 \mathrm{~K}$ and a parameterization valid over this range. We adopted a single linear function at each initial temperature over the entire range of particle velocities considered. Total uncertainties of all the EOS parameters and correlation coefficients for these uncertainties are given. The improved predictive capabilities of our EOS for Mo are confirmed by (1) better agreement between calculated bulk sound speeds and published measurements along the principal Hugoniot, (2) good agreement between our Grüneisen data and three reported high-pressure $\gamma(V)$ functions obtained from shock-compression of porous samples, and (3) very good agreement between our 1 bar Grüneisen values and $\gamma(T)$ at ambient pressure recalculated from reported experimental data on the adiabatic bulk modulus $K_{s}(T)$. Our analysis shows that an EOS constructed from shock compression data allows a much more accurate prediction of $\gamma(T)$ values at 1 bar than those based on static compression measurements or first-principles calculations. Published calibrations of the Mie-Grüneisen EOS for Mo using static compression measurements only do not reproduce even low-pressure asymptotic values of $\gamma(T)$ at 1 bar, where the most accurate experimental data are available. Published by AIP Publishing. [http://dx.doi.org/10.1063/1.4978607]
\end{abstract}

\section{INTRODUCTION}

In all our shock experiments on hot $\mathrm{MgO}$ preheated to initial temperatures of 1850 or $2300 \mathrm{~K}$, the samples were heated in molybdenum capsules that also served as driver plates. ${ }^{1,2}$ Analysis of data from these experiments requires an accurate knowledge of the Hugoniot and sound speed for molybdenum shock-compressed from elevated initial temperatures to ca. 100-500 GPa. In particular, our previous report noted a systematic $6 \%$ to $8 \%$ discrepancy between the sound speed in the shock state predicted for $\mathrm{MgO}$ pre-heated to $2300 \mathrm{~K}$ and that estimated from our experiments. ${ }^{2}$ Since the rarefaction wave used to determine the sound speed must travel through the hot Mo driver before entering the $\mathrm{MgO}$ sample, one suspected reason for this discrepancy is an inaccurate equation of state (EOS) for hot Mo.

The considerable scatter among the best estimated principal Hugoniots and Grüneisen coefficients at ambient conditions for molybdenum reported in the literature ${ }^{3-8}$ and the incorporation in the previous analysis of the hot Mo Hugoniot $^{9}$ of an assumed bcc-hcp solid-solid phase transition $^{8}$ that is no longer supported by data ${ }^{7,10}$ motivated our reanalysis of available room-temperature and $1673 \mathrm{~K}$ data. A further motivation arose from the absence of information in any reported EOS for $\mathrm{Mo}^{3,5-8}$ on the uncertainties in the ambient Grüneisen values or on the correlation between the uncertainties of Hugoniot parameters and Grüneisen

\footnotetext{
a)fatyan1@gps.caltech.edu

b)asimow@gps.caltech.edu
}

functions. This greatly complicated error analysis for the maximum likelihood prediction of Mo Hugoniots with 1850 or $2300 \mathrm{~K}$ initial temperatures.

This paper is organized as follows: The results of our analysis of reported experimental Hugoniot data for Mo shocked from ambient $T$ are summarized in Section II. The selection of the Grüneisen model and construction of the maximum likelihood linear Hugoniot for initial temperature of $1673 \mathrm{~K}$, the only elevated temperature at which data are available, are described in Section III. Section IV describes the algorithms for error analysis and the results of our parameterized EOS for Mo with initial temperatures from 298 to 2350 K. Section V demonstrates predictive capabilities of our EOS for the experimental parameters reported by other groups but not used to construct the current EOS: bulk sound speed in shock states lying along the ambient- $T$ Hugoniot, $\gamma(V)$ from ambient volume $V_{0}$ to $V=0.6 \times V_{0}$, and $\gamma(T)$ at 1 bar. The advantages of our new EOS and issues with popular EOS models for Mo constructed from static data alone are discussed in Section VI.

\section{PRINCIPAL HUGONIOT}

For the analysis of the principal Hugoniot (i.e., the family of shock states obtained from Mo initially at room- $T$ ) we selected the data from Refs. 4 (p. 108), 6, 8, and 11-14. Mo is known to exhibit an elastic-plastic double-wave structure at low stress. ${ }^{15}$ Therefore, we excluded from our analysis all data points with particle velocities $(U)$ below $\sim 0.95 \mathrm{~km} / \mathrm{s}$ or shock velocities $(D)$ below $\sim 6.5 \mathrm{~km} / \mathrm{s}$. The highest pressure 
data points included were two light-gas gun results of Mitchell et al. ${ }^{14}$ at $U$ of $\sim 4.3$ and $\sim 4.4 \mathrm{~km} / \mathrm{s}$. All the data from their underground nuclear tests were excluded. The accurate high-pressure data points of Al'tshuler et al. ( $U$ of 6.59 and $7.05 \mathrm{~km} / \mathrm{s})^{16}$ and Trunin et al. $(U=8.58 \mathrm{~km} / \mathrm{s})^{17}$ were not included because they exceeded the highest Mo particle velocity in our experiments by a factor of 1.5-2. Like other metals, Mo exhibits some noticeable nonlinearity of the Hugoniot slope at that pressure level ( $>1 \mathrm{TPa}) .{ }^{17}$

Since only two data points of Mitchell et al. ${ }^{14}$ had uncertainties for $U$ and $D$ reported by the authors, we assigned $1 \%$ relative uncertainties for all other data, as was done for a similar analysis of Hugoniots by Mitchell and Nellis ${ }^{18}$ or Kalitkin and Kuz'mina. ${ }^{19}$ We also considered errors for $U_{i}$ and $D_{i}$ at each data point $i$ to be correlated with a correlation coefficient of -0.85 , typical for this type of measurement (see Section I A of the supplementary material). Errors from different $\left(U_{i}, D_{i}\right)$ points were treated as uncorrelated. We used the main algorithm of the most general least-squares fitting procedure (Böck and Brandt) ${ }^{20,21}$ explained in detail only for the particular case of fitting $\left(x_{i}, y_{i}\right)$ data to the $y=a \times x$ linear function by Heiles in the early version of his unpublished report. ${ }^{22}$ Of about 10 different least-squares algorithms that we tried, it was the only method that correctly handled $\left(x_{i}, y_{i}\right)$ data with uncertainties in both coordinates (Section I B of the supplementary material). All the key equations we employed for the data analysis were derived from the maximum likelihood principle and can be found in Section I C of the supplementary material.

The validity of each linear fit was checked by first fitting $D$ as a function of $U$ and then fitting $U$ as a function of $D$, taking the inverse function, and comparing the results. We argue that any objective fitting routine should yield, within uncertainty, identical results for these two fits. In fact, with the method selected, all parameters (intercepts, slopes, their uncertainties, and the correlation between these uncertainties) for the $D$ as a function of $U$ Hugoniot by the direct and inverse fits were nearly identical. The maximum absolute difference was in the 3 rd significant digit or 5 times smaller than corresponding uncertainties of the fit parameters (see Table I). Hence, our linear regressions for $D$ vs. $U$ Hugoniots

TABLE I. Comparison of the maximum likelihood principal Hugoniots for Mo obtained from the $D$ vs. $U$ and $U$ vs. $D$ fits. Insignificant digits are shown to emphasize the differences. The opposite sign of the absolute slope and intercept differences confirms the strong anti-correlation of the best linear fit parameters. The reduced misfit was calculated for $n=58$ data points and $q=2$ parameters or $(n-q)=56$ degrees of freedom.

\begin{tabular}{lccc}
\hline \hline Parameter & D vs. U fit & U vs. D fit & $\begin{array}{c}\text { Absolute } \\
\text { difference }\end{array}$ \\
\hline Intercept, $a$ & 5.09177 & 5.08821 & 0.00356 \\
Intercept uncertainty, $\sigma_{a}$ & 0.017484 & 0.017498 & $1.5 \times 10^{-5}$ \\
Slope, $b$ & 1.26413 & 1.26582 & -0.00169 \\
Slope uncertainty, $\sigma_{b}$ & 0.0078127 & 0.0078199 & $7.2 \times 10^{-6}$ \\
Uncertainty correlation, & -0.94275 & -0.94254 & $2.1 \times 10^{-4}$ \\
cor $\left(\sigma_{a}, \sigma_{b}\right)$ & & & \\
Absolute reduced misfit, & 0.262710 & 0.262813 & $1.03 \times 10^{-4}$ \\
$\chi^{2} /(n-q)$ & & & \\
\hline \hline
\end{tabular}

are maximum likelihood solutions, independent of the choice of dependent variable. Treating uncertainties of $U_{i}$ and $D_{i}$ at each data point as uncorrelated did not change the slope and intercept by more than $+3.7 \times 10^{-4} \approx 0.047 \times \sigma_{b}$ and -8.2 $\times 10^{-4} \approx 0.047 \times \sigma_{a}$, respectively.

Our best fit Hugoniot for room- $T$ Mo (Fig. 1, lower panel) is $D=5.092( \pm 0.018)+1.264( \pm 0.008) \times U$, with a correlation coefficient of -0.94 between the uncertainties in the slope and intercept. This single linear dependence, valid in the $U$ range from 0.99 to $4.4 \mathrm{~km} / \mathrm{s}$, is quite consistent with the $D=(5.100 \pm 0.017)+(1.266 \pm 0.006) \times U$ relationship ${ }^{16,17}$ reported for $U<8.6 \mathrm{~km} / \mathrm{s}$ and deviates by only $0.09 \%-0.5 \%$ from the low-pressure asymptote, valid at $U<4.5 \mathrm{~km} / \mathrm{s}, D=(5.127 \pm 0.008)+(1.243 \pm 0.006) \times U$ $+8.34 \times 10^{-5} \times U^{2}$, of the wide-range curve of Kalitkin and Kuz'mina. ${ }^{19}$ In the $U$ range from 2 to $4 \mathrm{~km} / \mathrm{s}$ relevant to the present experiments, the uncertainty in Mo shock velocity does not exceed $\pm 0.015 \mathrm{~km} / \mathrm{s}$ at the $1 \sigma$ level. For comparison, the maximum difference between $D$ values calculated using the two best-fit expressions in Table I over the same $U$ range is only $0.004 \mathrm{~km} / \mathrm{s}$.

\section{III. $1673 \mathrm{~K}$ HUGONIOT FROM FITS TO EXPERIMENTAL DATA}

\section{A. Selection of the Grüneisen model}

Analysis of preheated Mo data obtained in our laboratory ${ }^{9,23,24}$ was done in several steps. In the following description, $\left(U_{1}, D_{1}\right)$ refers to a point on the room temperature

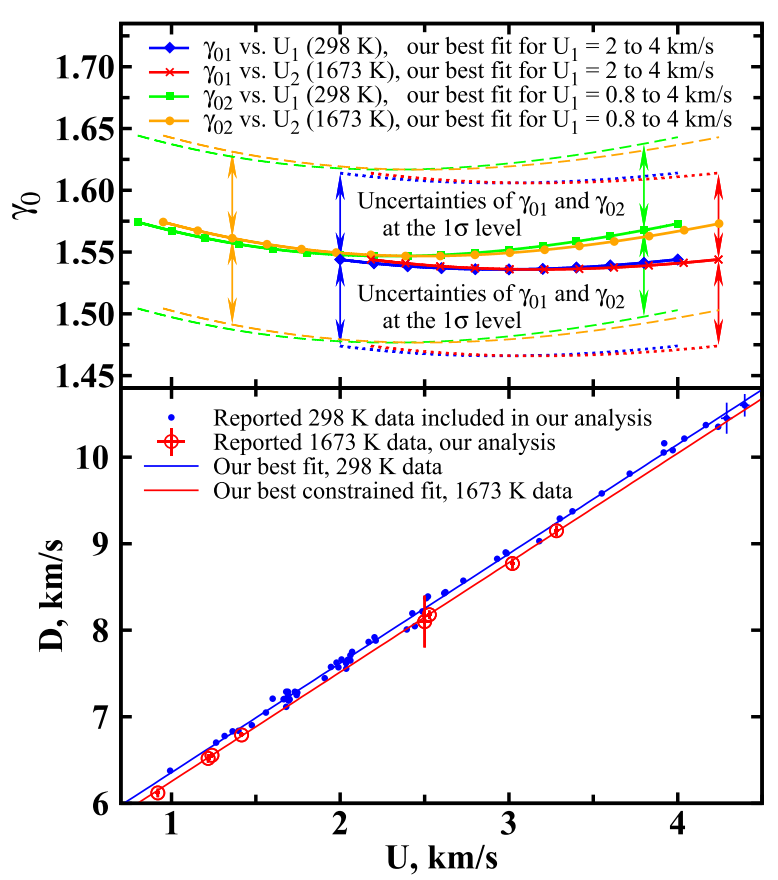

FIG. 1. Maximum likelihood fits to $298 \mathrm{~K}$ and updated $1673 \mathrm{~K} D_{2}$ vs. $U_{2}$ data for Mo (lower panel) and analytically computed values of apparent $\gamma_{0}$ from the double-constrained fits to experimental $1673 \mathrm{~K}\left(U_{2 i}, D_{2 i}\right)$ data optimized for two different ranges of $U_{1}$ on the $298 \mathrm{~K}$ Hugoniot (upper panel). $D_{21}=4.98924+1.26352 \times U_{2}$ and $D_{22}=4.98668+1.26496 \times U_{2}$ hot Hugoniots were used to compute $\gamma_{01}$ (for $U_{1}$ from 2.0 to $4.0 \mathrm{~km} / \mathrm{s}$ ) and $\gamma_{02}$ (for $U_{1}$ from 0.8 to $4.0 \mathrm{~km} / \mathrm{s}$ ), respectively. Note the particle velocity (horizontal) offset between the matching $\gamma_{0}\left(U_{1}\right)$ and $\gamma_{0}\left(U_{2}\right)$ curves and its monotonic increase with $U$. All crosses indicate the actual error bars. 
principal Hugoniot of Mo, whereas $\left(U_{2}, D_{2}\right)$ refers to the corresponding point at an equal volume on the preheated $1673 \mathrm{~K}$ Hugoniot. First, we rejected from the analysis all low stress data that showed a two-wave elastic-plastic response. ${ }^{24}$ Then, we slightly updated the remaining 8 hot Mo data points as outlined below. Our new room- $T$ Hugoniot was used to calculate the particle velocity values and their uncertainties for all experiments that employed Mo impactors (shots \# 355, 356, 360 , and 362). ${ }^{9}$ For the remaining four experiments, we first removed insignificant digits from the velocities measured in hot Mo $\left(D_{2}\right.$ for shots \# 716, 725, and 741 with $\mathrm{W}$ impactors ${ }^{23}$ and $U_{2}$ for shot \# 868 with Ta impactor ${ }^{24}$ ) and their respective uncertainties. Then, we updated, by standard shock impedance matching, the values of $U_{2}$ for shots \# 716, 725, and 741 and $D_{2}$ for shot \# 868 and computed new uncertainties by a conventional Monte Carlo procedure, similar to one outlined in the supplement of Ref. 25.

Next, we fitted these 8 hot Mo data points with $D_{2}>$ $6.1 \mathrm{~km} / \mathrm{s}$ to a linear function via the same procedure as described in Section II and computed an apparent reference Grüneisen parameter $\gamma_{0}$ at each point from the offset of the room- $T$ and hot Hugoniots assuming a Mie-Grüneisen approximation, a provisional constant $(\gamma / V)$ model, and estimates for the density and internal energy of Mo at ambient pressure. The density of Mo at $1673 \mathrm{~K}$ was calculated from the reported thermal expansion data. ${ }^{26-28}$ The difference of specific internal energies of Mo between room- $T$ and $1673 \mathrm{~K}$ was calculated from the specific heat at constant pressure. ${ }^{28-30}$ The uncertainties of density and specific internal energy difference at any particular temperature were evaluated as root-mean square deviations about the average values among the three sources for each quantity, assuming uncorrelated data. Full uncertainties took into account variations of thermodynamic parameters with the initial temperature, which is known to $\pm 3 \mathrm{~K}$. ${ }^{9}$ Since this is an unconstrained fit of $\left(U_{2}, D_{2}\right)$ data without any relationship to $\left(U_{1}, D_{1}\right)$ data, it is not forced to yield a consistent value of the nominally constant parameter $\gamma_{0}$. In fact, it yields a range for $\gamma_{0}$ from 1.540 at $U_{1}=2.0 \mathrm{~km} / \mathrm{s}$ to 1.504 at $U_{1}=4.0 \mathrm{~km} / \mathrm{s}$. This preliminary fit is shown as the first row of Table II.

Now, we tried to derive a more consistent overall fit that adopts some systematic behavior of the Grüneisen function. We performed constrained fits of the $1673 \mathrm{~K}$ Hugoniot data to the slightly non-linear $D_{2}$ vs. $U_{2}$ functions that result from an offset from the principal Hugoniot using a Mie-Grüneisen EOS and the three most popular models for the volume dependence of $\gamma^{5,31,32}$ Each of these models, assuming a linear room- $T$ reference Hugoniot, produces a somewhat nonlinear high- $T$ Hugoniot. For each model, the value of $\gamma_{0}$ was fitted simultaneously with iterative computation of maximum likelihood estimates $\left(U_{2 i}^{\#}, D_{2 i}^{\#}\right)$ for the experimental $\left(U_{2 i}, D_{2 i}\right)$ data until a good match was obtained with the simulated hot Hugoniot.

Then, these three sets of the intermediate maximum likelihood $\left(U_{2 i}^{\#}, D_{2 i}^{\#}\right)$ data were fitted to three straight lines via the same general procedure outlined above. This second fitting yielded the final maximum likelihood $\left(U_{2 i}^{*}, D_{2 i}^{*}\right)$ data for each $\gamma(V)$ model. Several reduced $\chi^{2}$ parameters were calculated using the following expressions:

$$
\begin{aligned}
& \chi_{1}^{2}=\frac{1}{n-q} \sum_{i=1}^{n}\left[\left(\frac{D_{2 i}^{\#}-D_{2 i}}{\sigma_{D_{2 i}}}\right)^{2}+\left(\frac{U_{2 i}^{\#}-U_{2 i}}{\sigma_{U_{2 i}}}\right)^{2}\right], \\
& \chi_{2}^{2}=\frac{1}{n-q} \sum_{i=1}^{n}\left[\left(\frac{D_{2 i}^{*}-D_{2 i}^{\#}}{\sigma_{D_{2 i}}}\right)^{2}+\left(\frac{U_{2 i}^{*}-U_{2 i}^{\#}}{\sigma_{U_{2 i}}}\right)^{2}\right], \\
& \chi^{2}=\frac{1}{n-q} \sum_{i=1}^{n}\left[\left(\frac{D_{2 i}^{*}-D_{2 i}}{\sigma_{D_{2 i}}}\right)^{2}+\left(\frac{U_{2 i}^{*}-U_{2 i}}{\sigma_{U_{2 i}}}\right)^{2}\right],
\end{aligned}
$$

where $n$ is the total number of data points ( $n=8$ for Mo), $q$ is the number of fitting parameters $\left(q=1\right.$ for all $\chi_{1}^{2}, q=2$ for all $\chi_{2}^{2}$, and $q=3$ for all $\chi^{2}$ except for the unconstrained linear fit shown in the first row of Table II where $q=2$ ), $U_{2 i}$ and $D_{2 i}$ are the measured values at the $i$-th point with $\sigma_{U_{2 i}}$ and $\sigma_{D_{2 i}}$ respective uncertainties, $U_{2 i}^{\#}$ and $D_{2 i}^{\#}$ are the maximum likelihood estimates of experimental data fitted to the Grüneisen models only, and $U_{2 i}^{*}$ and $D_{2 i}^{*}$ are the final maximum likelihood estimates of the $D_{2 i}^{\#}$ vs. $U_{2 i}^{\#}$ data (rows \#2-4 of Table II) or of the measured $D_{2 i}$ vs. $U_{2 i}$ data (rows \#1 and \#5 of Table II) fitted to linear functions. To be clear, $\chi_{1}^{2}$ represents the misfit between the experimental data and the most likely corresponding points along the non-linear high- $T$ Hugoniot obtained by a Mie-Grüneisen offset from the principal Hugoniot; $\chi_{2}^{2}$ represents the misfit between those points

\begin{tabular}{|c|c|c|c|c|c|}
\hline Constraint & $\gamma_{0}$ & $\chi_{1}^{2}$ & $D$ to $U$ linear fit & $\chi_{2}^{2}$ & $\chi^{2}$ \\
\hline$\cdots$ & $1.540-1.504$ & $\cdots$ & $4.993(14)+1.262(9) \times U$ & $\cdots$ & 0.167 \\
\hline$\gamma=\gamma_{0} \times V / V_{0}{ }^{\mathrm{a}}$ & 1.564 & 0.177 & $4.9848(9)+1.2664(6) \times U$ & $0.7 \times 10^{-3}$ & 0.208 \\
\hline$\gamma=\frac{2}{3}+\left(\gamma_{0}-\frac{2}{3}\right) \times\left(V / V_{0}\right)^{\frac{\gamma_{0}}{\gamma_{0}^{-2 / 3}} \mathrm{~b}}$ & 1.537 & 0.207 & $4.9759(12)+1.2725(7) \times U$ & $1.2 \times 10^{-3}$ & 0.245 \\
\hline$\gamma=\frac{2}{3}+\frac{2 V}{\left[1+2 /\left(\gamma_{0}-2 / 3\right)\right] \times V_{0}-V} c$ & 1.469 & 0.255 & $4.967(2)+1.2785(13) \times U$ & $3.8 \times 10^{-3}$ & 0.312 \\
\hline$\gamma=\gamma_{0} \times V / V_{0}$ and $D=c+s \times U$ & 1.536 to 1.544 & $\ldots$ & $4.98924+1.26352 \times U$ & $\ldots$ & 0.202 \\
\hline
\end{tabular}
along the non-linear high- $T$ model Hugoniot and

TABLE II. Comparison of 1673 K Mo Hugoniot fits for different Grüneisen models.

${ }^{\mathrm{a}}$ References 3 and 31 (p. 297).

${ }^{\mathrm{b}}$ Reference 5 .

${ }^{c}$ References 32 and 33. 
corresponding most likely points along a linear high- $T$ Hugoniot; and finally, $\chi^{2}$ represents the misfit between the original high- $T$ Hugoniot data and the most likely points along the linear model high- $T$ Hugoniots. No intermediate maximum likelihood values were obtained for the conventional unconstrained and double-constrained fits (see below) shown in rows \#1 and \#5 of Table II, respectively.

The results of this analysis, summarized in Table II, clearly indicate that the best fit results from the simplest $\gamma(V)=\gamma_{0} \times V / V_{0}$ model, at least for the narrow range of parameters relevant to our study (particle velocity $U_{1}$ on the room- $T$ Hugoniot ranging from 2.0 to $4.0 \mathrm{~km} / \mathrm{s}$ ). This model gave the lowest value of the reduced $\chi^{2}$ and the best agreement between the maximum likelihood value of the ambient Grüneisen coefficient and that derived from known thermodynamic parameters of Mo. Therefore, in subsequent analysis, we restricted the choice of Grüneisen coefficient models to the one best supported by our data, $\gamma(V)=\gamma_{0} \times V / V_{0}$.

\section{B. Maximum likelihood linear $D_{2}$ vs. $U_{2}$}

Finding the maximum likelihood parameters of a linear hot Hugoniot is straightforward for the initial temperature of $1673 \mathrm{~K}$, where the actual $D_{2}$ vs. $U_{2}$ data exist. This is done as described in Section II B of the supplementary material using a double-constrained fit of hot Hugoniot data points to a linear $D_{2}$ vs. $U_{2}$ dependence (1st constraint) optimized before fitting to satisfy the $\gamma(V)=\gamma_{0} \times V / V_{0}$ Grüneisen model (2nd constraint). This newly constructed Hugoniot was then applied to the analysis of shot $\# 868^{24}$ to update the shock velocity in hot Mo. The double-constrained fit was then repeated for the hot Mo dataset with one updated point. Since it did not noticeably change the best fit parameters, no further iterations were done. The results of this regression are included for comparison in Table II and indicate a small increase in the reduced $\chi^{2}$ values compared to the first unconstrained linear fit but a smaller range of apparent ambient Grüneisen parameters and a closer fit to the $\gamma_{0}$ predicted from measured thermodynamic parameters.

The lowest value of $U_{1}=2 \mathrm{~km} / \mathrm{s}$ limited the highest shock volume on the room- $T$ Hugoniot which, in turn, puts 4 experimental hot Mo Hugoniot points with $U_{2} \leq 1.5 \mathrm{~km} / \mathrm{s}$ outside the range of shock volumes accessible from both $298 \mathrm{~K}$ and $1673 \mathrm{~K}$ Hugoniots. In other words, these points were used to constrain the linear fit and reduce the parameter uncertainties for the $1673 \mathrm{~K}$ Hugoniot only but were not immediately used to constrain $\gamma$. Therefore, we performed a consistency check for the best fit values of $\gamma_{0}$ determined that way by extending the lower limit of $U_{1}$ to $0.8 \mathrm{~km} / \mathrm{s}$. This increased the upper limit of shock compressed volume on the room- $T$ Hugoniot and allowed all 8 hot Mo data points to have the same values of shocked volume as those generated on the $298 \mathrm{~K}$ Hugoniot over this $U_{1}$ range. In this procedure, we used the same best fit parameters for the linear room- $T$ Mo Hugoniot as before. This means that we were not adding any data points with a two-wave structure to the analysis but rather extrapolated our high-pressure part of the reference (room- $T$ ) Hugoniot to the required low-pressure range. The results of this analysis for the $U_{1}$ range from 0.8 to $4.0 \mathrm{~km} / \mathrm{s}$ yielded $\gamma_{0}=1.557, b=1.264964$, and $a=4.986679$.

The originally reported Hugoniot data for $1673 \mathrm{~K}$ Mo and the results of our analysis are summarized in Table III. All experimental room- $T D_{1}$ vs. $U_{1}$ and updated $1673 \mathrm{~K} D_{2}$ vs. $U_{2}$ Hugoniot data for Mo are shown in Figure 1 (lower panel) along with the best fit apparent $\gamma_{0}$ values (upper panel). It should be emphasized that preheated Mo in most shock experiments is compressed from the solid phase but ends in the liquid. ${ }^{9}$ Therefore, our analysis gives only an apparent value of the thermal Grüneisen coefficient relevant to this particular range of conditions. Only in the particular volume range relevant to our studies does the $\gamma(V)=\gamma_{0}$ $\times V / V_{0}$ model work better than other known advanced and more sophisticated models. For the wide range EOS, it will be mandatory to use more advanced realistic Grüneisen models $^{34}$ instead of $\gamma(V)=\gamma_{0} \times V / V_{0}$.

\section{HOT HUGONIOTS PREDICTED BY OUR EOS}

For the initial temperatures of interest for our $\mathrm{MgO}$ experiments, $1850 \mathrm{~K}$ and $2300 \mathrm{~K}$, the response of Mo to shock loading has not been characterized directly. Instead, the corresponding hot Hugoniots need to be constructed as offsets from the room- $T$ Hugoniot using the $1673 \mathrm{~K}$ constraints and a suitable model. There are several parameters

TABLE III. Summary of experimental Hugoniot data for $1673 \mathrm{~K}$ molybdenum.

\begin{tabular}{|c|c|c|c|c|c|c|}
\hline \multirow[b]{2}{*}{ Shot \# } & \multicolumn{3}{|c|}{ Shock velocity } & \multicolumn{3}{|c|}{ Particle velocity } \\
\hline & $\begin{array}{l}\text { Reported, } \\
D_{0}(\mathrm{~km} / \mathrm{s})\end{array}$ & $\begin{array}{l}\text { Revised, } \\
D(\mathrm{~km} / \mathrm{s})\end{array}$ & $\begin{array}{l}\text { Model difference, } \\
\quad\left(D^{\star}-D\right) / \sigma_{D}\end{array}$ & $\begin{array}{l}\text { Reported, } \\
U_{0}(\mathrm{~km} / \mathrm{s})\end{array}$ & $\begin{array}{l}\text { Revised, } \\
U(\mathrm{~km} / \mathrm{s})\end{array}$ & $\begin{array}{l}\text { Model difference, } \\
\quad\left(U^{\star}-U\right) / \sigma_{U}\end{array}$ \\
\hline $716^{\mathrm{a}}$ & $6.115(47)$ & $6.12(5)$ & 0.60 & $0.919(4)$ & $0.919(5)$ & -0.07 \\
\hline $725^{\mathrm{a}}$ & $6.516(49)$ & $6.52(5)$ & 0.14 & $1.219(9)$ & $1.217(8)$ & -0.02 \\
\hline $868^{\mathrm{b}}$ & $6.549(35)$ & $6.556(16)$ & 0.00 & $1.238(14)$ & $1.240(12)$ & 0.00 \\
\hline $741^{\mathrm{a}}$ & $6.788(21)$ & $6.79(2)$ & -0.51 & $1.414(6)$ & $1.416(6)$ & 0.18 \\
\hline $355^{\mathrm{c}}$ & $8.123(300)$ & $8.1(3)$ & 0.16 & $2.495(40)$ & $2.50(4)$ & -0.03 \\
\hline $356^{\mathrm{c}}$ & $8.180(53)$ & $8.18(5)$ & 0.07 & $2.522(16)$ & $2.528(8)$ & -0.01 \\
\hline $362^{c}$ & $8.768(58)$ & $8.77(6)$ & 0.58 & $3.011(10)$ & $3.021(11)$ & -0.12 \\
\hline $360^{\mathrm{c}}$ & $9.149(57)$ & $9.15(6)$ & -0.22 & $3.270(22)$ & $3.282(10)$ & 0.04 \\
\hline
\end{tabular}

${ }^{\mathrm{a}}$ Reference 23.

${ }^{\mathrm{b}}$ Reference 24 .

${ }^{\mathrm{c}}$ Reference 9. 
TABLE IV. Representative Hugoniots for Mo, $D=a+b \times U$, predicted by our EOS. The first two lines are fits to the experimental data. Numbers in parentheses are $1 \sigma$ uncertainties for the last significant digit(s).

\begin{tabular}{lccccc}
\hline \hline$T_{0}(\mathrm{~K})$ & $\rho_{0}(\mathrm{~g} / \mathrm{cm})^{3}$ & $\Delta E_{0}(\mathrm{~J} / \mathrm{g})$ & $a\left(\sigma_{a}\right)(\mathrm{km} / \mathrm{s})$ & $b\left(\sigma_{b}\right)$ & $\operatorname{cor}\left(\sigma_{a}, \sigma_{b}\right)$ \\
\hline $298(5)$ & $10.205(5)$ & $\ldots$ & $5.092(18)$ & $1.264(8)$ & $-0.94(3)$ \\
$1673(3)$ & $9.940(6)$ & $408.0(14)$ & $4.989(14)$ & $1.264(9)$ & $-0.93(3)$ \\
& & & & & \\
1673 & $9.940(5)$ & $408.0(10)$ & $4.99(2)$ & $1.264(9)$ & $-0.99(3)$ \\
1850 & $9.897(5)$ & $470.7(11)$ & $4.97(2)$ & $1.264(9)$ & $-0.98(3)$ \\
2300 & $9.771(5)$ & $646.9(17)$ & $4.92(3)$ & $1.264(10)$ & $-0.98(3)$ \\
\hline \hline
\end{tabular}

involved in the nonlinear transformations from a room- $T D_{1}$ vs. $U_{1}$ Hugoniot to a predicted high temperature Hugoniot. The construction of a maximum likelihood hot Hugoniot requires accurate knowledge of the uncertainties and multivariate correlations between the uncertainties for all the parameters involved in the transformation.

The uncertainty of $\gamma_{0}$ and its correlation with the uncertainties of other essential parameters (slope and intercept of room- $T$ Hugoniot, densities of ambient and hot Mo, and internal energy difference between the ambient and hot Mo) were calculated by a conventional Monte Carlo simulation procedure (Section III of the supplementary material) similar to one outlined in the supplementary material of Ref. 25.

We generated complete sets of parameters and their correlated uncertainties for 21 values of $U_{1}$ equally spaced across our primary range of interest, from 2.0 to $4.0 \mathrm{~km} / \mathrm{s}$, corresponding to a range of $U_{2}$ on the $1673 \mathrm{~K}$ Hugoniot from 2.18 to $4.24 \mathrm{~km} / \mathrm{s}$. The highest values of $\gamma_{0}=1.544$ were obtained at the ends of range, $U_{1}$ of 2.0 and $4.0 \mathrm{~km} / \mathrm{s}$; the lowest values of 1.536 were in the center of the range at $U_{1}$ of 2.9 and $3.0 \mathrm{~km} / \mathrm{s}$ (Fig. 3, upper panel). These data were used later to generate synthetic hot Mo Hugoniots for initial temperatures relevant to our experiments: 1673,1850 , and $2300 \mathrm{~K}$.

As a consistency check, we first generated a synthetic Mo Hugoniot for the initial temperature of $1673 \mathrm{~K}$, where data are available. Details can be found in Section IV of the supplementary material. Comparison of the best fit parameters listed in the second and third lines of Table IV indicates a very good agreement between the direct analysis and synthetic Hugoniots for Mo with the initial temperature of $1673 \mathrm{~K}$. Similar routines were applied to the same initial sets of correlation coefficients to generate synthetic Mo Hugoniots for initial temperatures of 1850 and $2300 \mathrm{~K}$. Corresponding best fit parameters and the initial thermodynamic values are also listed in Table IV.

We also parameterized a complete set of synthetic Hugoniot parameters applicable to initial temperatures from 298 to $2350 \mathrm{~K}$. The best fit expressions for the initial densities, slopes, and intercepts; their uncertainties; correlations of uncertainties; and the applicable ranges of particle velocities at each temperature are summarized in Table V.

\section{VALIDATION OF OUR EOS AND GRÜNEISEN MODEL}

\section{A. Prediction of bulk sound speed values above $400 \mathrm{GPa}$}

To validate our choice of the room- $T$ Hugoniot and Grüneisen model, we computed the bulk sound speed values at four points that match three experimental data points reported in Ref. 8 and one point reported in Ref. 10. The parameters for Mo shock-compressed by Ta impactors were evaluated using the reported Hugoniot for $\mathrm{Ta}^{18}$ and the principal Hugoniot of Mo obtained in this study. Particle velocities in molten Mo upon shock compression from room temperature are slightly higher than $4.0 \mathrm{~km} / \mathrm{s}$, the upper limit value for our Grüneisen model. Nevertheless, our predicted bulk sound speed values, listed in Table VI, are in good agreement with all reported experimental data.

Section V A of the supplementary material also lists the results of similar analyses incorporating the Mo Hugoniot

TABLE V. Summary of the best fit parameters of the Mo Hugoniot predicted by our EOS as a function of the initial temperature, $D(T)=a(T)+b(T) \times U$. All parameters are expressed as $Q(T)=k_{0}+k_{1} \times(T-298)+k_{2} \times(T-298)^{2}+k_{3} \times(T-298)^{3}$, where $T$ is the absolute temperature in K. ${ }^{\text {a }}$ Every individual Hugoniot is valid for its own range of particle velocities from $U^{\min }(T)$ to $U^{\max }(T)$. This range varies with the initial Mo temperature from 2.0 to $4.0 \mathrm{~km} / \mathrm{s}$ at $298 \mathrm{~K}$ to 2.3 to $4.4 \mathrm{~km} / \mathrm{s}$ at $2350 \mathrm{~K}$.

\begin{tabular}{lccc}
\hline \hline & \multicolumn{2}{c}{ Best fit coefficients } \\
\cline { 2 - 4 } Parameter & $k 0$ & $k 1$ & $k 2$ \\
\hline Initial density $\rho_{0}(T)(\mathrm{g} / \mathrm{cm})^{3}$ & 10.205 & $-1.635 \times 10^{-4}$ & $-9.787 \times 10^{-9}$ \\
Density uncertainty & 0.005 & $\ldots$ & $\ldots 3$ \\
$\sigma_{\rho_{0}}(T)(\mathrm{g} / \mathrm{cm})^{3}$ & 5.092 & $-6.184 \times 10^{-5}$ & $-5.897 \times 10^{-9}$ \\
Intercept $a(T)(\mathrm{km} / \mathrm{s})$ & $0.02(T<2250 \mathrm{~K})$ & $\ldots$ & $\ldots$ \\
Intercept uncertainty & $0.03(T \geq 2250 \mathrm{~K})$ & $\ldots$ & $\ldots$ \\
$\sigma_{a}(T)(\mathrm{km} / \mathrm{s})$ & 1.264 & $\ldots$ & $\ldots$ \\
Slope $b(T)$ & $0.009(T<2250 \mathrm{~K})$ & $\ldots$ & $\ldots$ \\
Slope uncertainty $\sigma_{b}(\mathrm{~T})$ & $0.010(T \geq 2250 \mathrm{~K})$ & $\ldots$ & $\ldots$ \\
Correlation of & $-0.96(3)$ & $1.114 \times 10^{-4}$ & $\ldots$ \\
uncertainties $\left(\sigma_{a}, \sigma_{b}\right)$ & 2.0 & $1.492 \times 10^{-4}$ & $\ldots$ \\
Lower limit $U^{\min }(T)(\mathrm{km} / \mathrm{s})$ & 4.0 & $\ldots .119 \times 10^{-9}$ & $\ldots$ \\
Upper limit $U^{\max }(T)(\mathrm{km} / \mathrm{s})$ & & $\ldots .152 \times 10^{-9}$ \\
\hline \hline
\end{tabular}

${ }^{\mathrm{a}}$ For example, the universal Hugoniot intercept from 298 to $2350 \mathrm{~K}$ is $a(T)=\left[5.092-6.184 \times 10^{-5} \times(T-298)-5.897 \times 10^{-9} \times(T-298)^{2}-3.056\right.$ $\left.\times 10^{-12} \times(T-298)^{3}\right] \mathrm{km} / \mathrm{s}$. 
TABLE VI. Comparison of reported sound speeds and those predicted by our EOS for molten Mo on the principal Hugoniot.

\begin{tabular}{lcclll}
\hline \hline \multirow{2}{*}{$U_{f}(\mathrm{~km} / \mathrm{s})$} & \multicolumn{2}{c}{$P(\mathrm{GPa})$} & & \multicolumn{2}{c}{$C_{b}(\mathrm{~km} / \mathrm{s})$} \\
\cline { 2 - 3 } \cline { 6 - 7 } & Experiment & Our model & & Experiment & Our model \\
\hline $7.14^{\mathrm{a}}$ & 402 & $402.5 \pm 1.5$ & & $9.33 \pm 0.17$ & $9.42 \pm 0.08$ \\
$7.36^{\mathrm{a}}$ & 421 & $421.6 \pm 1.6$ & & $9.42 \pm 0.17$ & $9.54 \pm 0.08$ \\
$7.58^{\mathrm{a}}$ & 441 & $441.1 \pm 1.7$ & & $9.62 \pm 0.17$ & $9.65 \pm 0.08$ \\
$7.625 \pm 0.002^{\mathrm{b}}$ & $438 \pm 8$ & $445.1 \pm 1.7$ & & $9.74 \pm 0.15$ & $9.67 \pm 0.08$ \\
\hline \hline
\end{tabular}

${ }^{\mathrm{a}}$ Reference 8 .

${ }^{\mathrm{b}}$ Reference 10 .

equations of state reported by other groups. ${ }^{3,5-9,33}$ While nearly all the EOS options reproduce the experimental shock pressures with good accuracy, our new EOS clearly shows better prediction of bulk sound speeds in shock-compressed Mo, with a reduced $\chi_{1}^{2}$ of 0.32 compared to 0.81 for the nextbest model. ${ }^{5}$

As seen in Figure 2, only our model demonstrates statistically significant overlap with all 4 experimental data points within their uncertainties. Other curves from seven different $\operatorname{EOS}^{3,5-9,33}$ plotted in Figure 2, except for the tabular $\gamma(V)$ model by Al'tshuler et al., ${ }^{5}$ apparently underestimate the sound speed in shocked molten Mo, at least in the 400 to $450 \mathrm{GPa}$ pressure range. Predictions by the model of Molodets ${ }^{33}$ clearly disagree with all the experimental data and other EOS values.

\section{B. Comparison with the reported empirical and analytical Grüneisen models for shock-compressed Mo}

Another possible validation of our Grüneisen model is direct comparison with the most accurate experimental values obtained and reported for Mo by other shock compression groups. ${ }^{3,5}$ A very good agreement was found between the values of the ambient Grüneisen coefficient obtained here $\left(\gamma_{0}=1.54 \pm 0.07\right)$ and those with unspecified

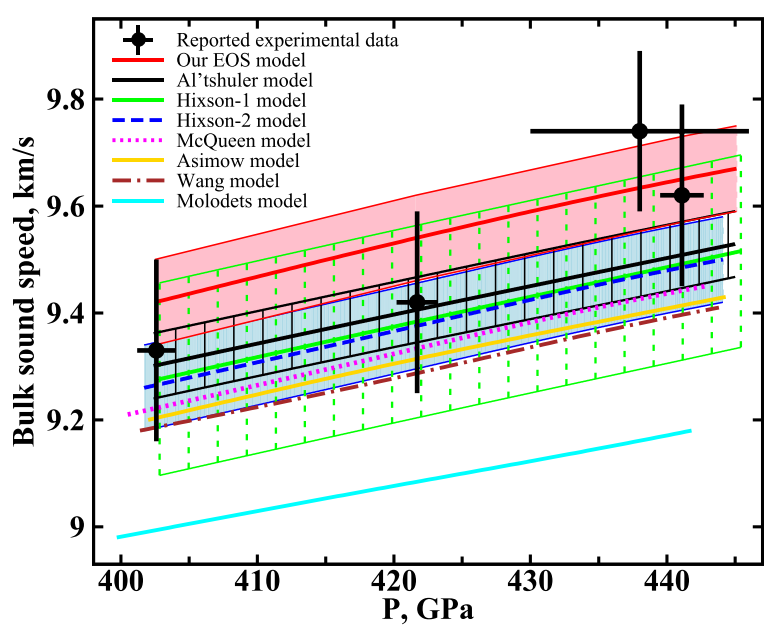

FIG. 2. Comparison of predicted sound speeds ${ }^{3,5-8,33}$ in Mo shocked above $400 \mathrm{GPa}$ with the reported experimental data. ${ }^{6,10}$ The upper and lower limits of Al'tshuler et al.'s EOS prediction plotted here correspond to Al'tshuler-2 (tabular data) and Al'tshuler-1 (formula) $\gamma(V)$ models, ${ }^{5}$ respectively. The shaded areas indicate uncertainties estimated by us. Crosses are the actual data error bars. uncertainties reported by McQueen et al. ${ }^{3}\left(\gamma_{0}=1.52\right)$ and Al'tshuler et al..$^{5}\left(\gamma_{0}=1.61\right)$. The ambient value recalculated from the $0 \mathrm{~K}$ parameters of the EOS of El'kin et al., ${ }^{36}$ $\gamma_{0}=1.63$, is just slightly outside the range of $1 \sigma$ uncertainty of our data. Since McQueen et al. adopted the same oneparameter functional form for $\gamma(V)$ as the one we adopt here, ${ }^{31}$ our result is in excellent agreement with McQueen et al. up to the highest volume compression considered (Figure 3). This conclusion remains valid regardless of the uncertainty level of their data.

The Grüneisen function of Al'tshuler et al. (the average of their formula and tabular data at equal compression) does deviate from the results of our model prediction at high pressure, with misfits ranging from $\sim 0.04$ at $U_{1} \approx 3.1 \mathrm{~km} / \mathrm{s}$ (i.e., $\left.V / V_{0} \approx 0.66\right)$ to $\sim 0.10$ at $U_{1}=4 \mathrm{~km} / \mathrm{s}\left(V / V_{0} \approx 0.61\right)$. This discrepancy is mainly caused by a different functional form for $\gamma(V)$ adopted by the authors of Ref. 5 and higher value of their ambient $\gamma_{0}$. However, this level of deviation is roughly equal to full systematic uncertainty of their Grüneisen function over that compression range. Our lower-limit error analysis (see Section V B of the supplementary material) gives absolute uncertainty of $\gamma$ from $\sim 0.09$ at $V / V_{0} \approx 0.66$ to $\sim 0.07$ at $V / V_{0} \approx 0.61$. With this uncertainty estimate, the results of Al'tshuler et al. ${ }^{5}$ agree with our data and those of McQueen et $a l^{3}$ over the entire range of compressions shown.

Finally, the maximum difference between the $\gamma(V)$ reported by El'kin et al. ${ }^{36}$ and our predictions over the intended valid range of our model $\left(0.74 \geq V / V_{0} \geq 0.61\right)$ is $\sim 0.08$ at $V / V_{0} \approx 0.74$. This level of discrepancy apparently does not exceed the level of uncertainty of experimental Grüneisen values discussed here. Comparison of our model values with an unweighted average of 3 reported functions for the maximum likelihood experimental data ${ }^{3,5,36}$ indicates that the difference never exceeds our $1 \sigma$ uncertainty at any volume compression range shown in Figure 3.

The reported empirical and semi-empirical Grüneisen functions were inferred from measurements on porous

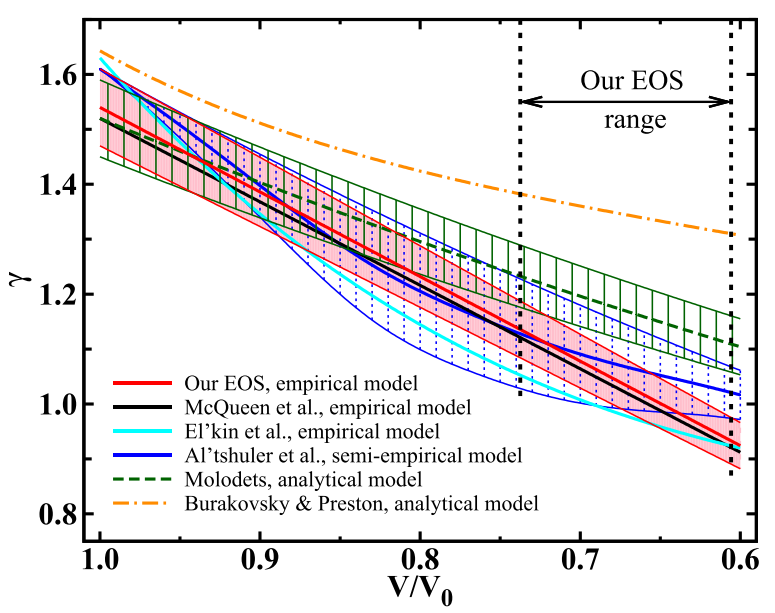

FIG. 3. Comparison of our $\gamma(V)$ model with other reported empirical, , $^{3,36}$ semi-empirical, ${ }^{5}$ and analytical models ${ }^{32,43}$ for shock-compressed Mo. The upper and lower limits of Al'tshuler et al.'s Grüneisen function correspond to Al'tshuler-2 (tabular data) and Al'tshuler-1 (formula) $\gamma(V)$ models, ${ }^{5}$ respectively. The shaded areas indicate uncertainties estimated by us. 
samples, which provide a large and easily-resolved difference from the Hugoniot of regular, full-density samples. Application of the state-of-the-art methods of statistical analysis allowed us to extract accurate Grüneisen data and get the same answer, within the estimated uncertainty, from the much smaller finite difference between the room- $T$ and $1673 \mathrm{~K}$ Hugoniots of full-density Mo (see Figure 1). Our result benefits also from being measured much closer to the temperature range of interest for shocks in condensed samples, which improves its accuracy in this area in the case that the Mie-Grüneisen approximation $\gamma=\gamma(V)$ is imperfect over wide temperature ranges.

Also shown for comparison in Figure 3 are two analytical Grüneisen models proposed recently for the description of shock-compressed solids. ${ }^{32,33,43}$ Uncertainties for the $\gamma(V)$ values of Molodets were estimated via a standard error propagation analysis for $\gamma_{0}$ only, assuming all other model parameters $\left(V\right.$ and $\left.V_{0}\right)$ to be exact. For the range of compressions relevant to our EOS, the model of Molodets ${ }^{33}$ agrees within the errorbars with the upper limit of the semi-empirical model of Al'tshuler et al. ${ }^{5}$ only. The model of Burakovky and Preston ${ }^{43}$ fails to predict any reported experimental Grüneisen values for shock-compressed Mo at least from $V / V_{0} \approx 0.8$ or above $\sim 95 \mathrm{GPa}$. As seen in Figure 3, our EOS model for $\gamma$ is consistent with all reported empirical or semi-empirical models but apparently does not support either of the proposed analytical models.

\section{Prediction of macroscopic $\gamma(\boldsymbol{T})$ at 1 bar}

The macroscopic Grüneisen coefficient $\gamma(T)$ at 1 bar can be computed (1) directly from the $\gamma(V)$ model

$$
\gamma_{1}(T)=\gamma_{0} \times V(T) / V_{0}=\gamma_{0} \times \exp \left(\int_{T_{0}}^{T} \alpha(T) d T\right)
$$

and (2) from the family of bulk sound speed values

$$
\begin{aligned}
\gamma_{2}(T) & =\alpha(T) \times V(T) \times K_{s}(T) / C_{p}(T) \\
& =\alpha(T) \times c_{0}(T)^{2} / C_{p}(T),
\end{aligned}
$$

where $\gamma_{0}=1.54 \pm 0.07$ is our model value, $\alpha(T)$ is the volume thermal expansion coefficient, $K_{s}(T)$ is the isentropic compression bulk modulus, $c_{0}(T)$ is the bulk sound speed, and $C_{p}(T)$ is specific heat (all parameters at $1 \mathrm{bar}$ ). For materials that (1) have negligible shear strength beyond the Hugoniot elastic limit and (2) do not exhibit phase transitions on shock compression, $c_{0}(T)$ is usually equal or almost equal to $a(T)$, the hot Hugoniot intercept. In this case, Equation (5) becomes

$$
\gamma_{2}(T)=\alpha(T) \times a(T)^{2} / C_{p}(T) .
$$

Molybdenum retains fairly low shear strength on shock compression, at least for initial temperatures of 298 and $1673 \mathrm{~K} .^{15,24}$ This property of Mo is also confirmed by very good agreement between the intercept of its room- $T$ Hugoniot, $5.092 \mathrm{~km} / \mathrm{s}$, and the values of bulk sound speed at ambient conditions, 5.041 to $5.124 \mathrm{~km} / \mathrm{s}$, recalculated from the reported adiabatic bulk modulus data. ${ }^{35}$ It would be very unlikely for a normal shock-loaded metal to exhibit shear strength increase at higher initial temperatures. This suggests negligible difference between the position of the Mo Hugoniot curve and a hydrostat ${ }^{15}$ (that is, the compression curve of a fluid with the same bulk modulus) and the same values of bulk sound speed at equal compression for the whole range of conditions relevant to the current study.

However, Mo melts on the $1673 \mathrm{~K}$ Hugoniot at any particle velocity in the range of validity of our EOS. ${ }^{9}$ The intercept of the $1673 \mathrm{~K}$ Hugoniot is $4.989 \mathrm{~km} / \mathrm{s}$, whereas the $1 \mathrm{bar}$ bulk sound speed at that temperature recalculated from the reported $K_{S}(T)$ data for solid $\mathrm{Mo}^{40}$ is only $4.817 \mathrm{~km} / \mathrm{s}$. This difference exceeds the level of estimated uncertainties by nearly an order of magnitude. Therefore, Equation (6) is only valid up to some threshold temperature lower than $1673 \mathrm{~K}$. This, in turn, suggests that any noticeable discrepancy between the values of $\gamma_{1}(T)$ and $\gamma_{2}(T)$ at $T \geqslant 1000 \mathrm{~K}$ does not indicate inconsistency in the current EOS.

We divide macroscopic Grüneisen $\gamma(T)$ curves at 1 bar available in the literature into those that are consistent, within uncertainties, with our model and those that are not. First, Figure 4 shows a comparison between our EOS prediction of $\gamma(T)$ and values calculated from experimental data on the temperature dependence of the Mo adiabatic bulk modulus $K_{s}(T) .{ }^{40-42}$ Only three sets of $\gamma(T)$ data are shown in Figure 4 as reported. ${ }^{37-39}$ All other $\gamma(T)$ curves or discrete data points were recalculated from the reported $K_{S}(T)$ experimental values ${ }^{40-42}$ or $\gamma(V)$ models $s^{3,5,6,36,43,44}$ using the same $\alpha(T)^{26-28}$ and $C_{p}(T)^{28-30}$ functions that we applied in constructing our Mo EOS.

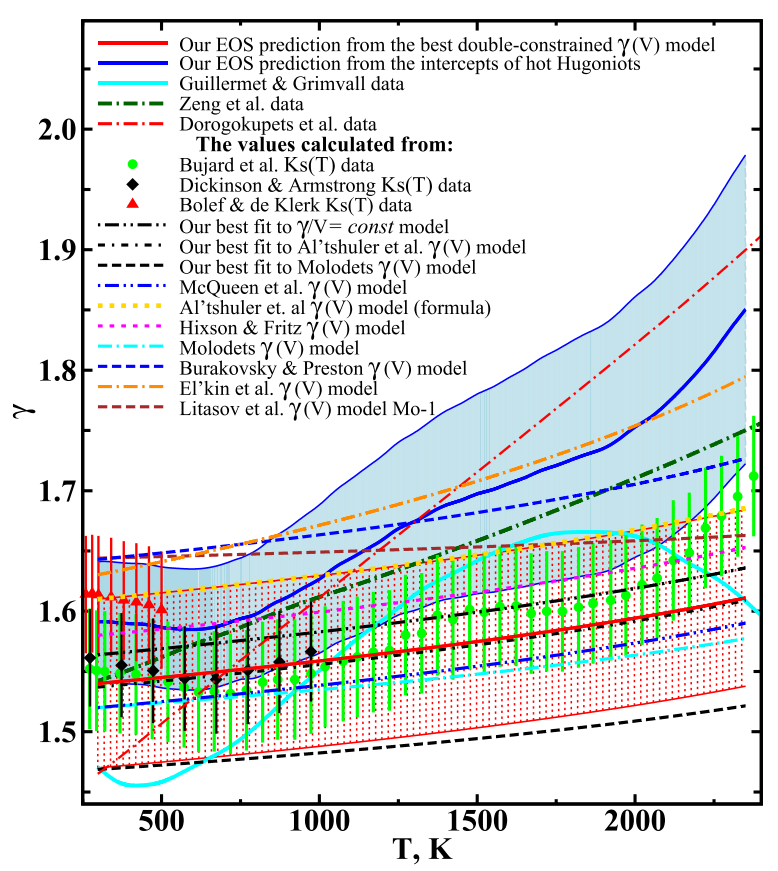

FIG. 4. Summary of macroscopic Grüneisen parameters at $1 \mathrm{bar}^{3,5,6,36-39,43,44}$ consistent with the results of our calculations from the reported measurements of $K_{s}(T)^{40-42}$ and the values predicted by our current EOS. Two shaded areas indicate the uncertainties of our $\gamma_{1}(T)$ (red) and $\gamma_{2}(T)$ (blue) data. Crosses at discrete data points are the estimated errorbars. 
Solid red and blue lines surrounded by shaded bands in Figure 4 are our $\gamma_{1}(T)$ and $\gamma_{2}(T)$ parameters and their uncertainties computed from Equations (4) and (6), respectively. All our primary macroscopic Grüneisen values, $\gamma_{1}(T)$, are in a very good agreement with the experimental data of Dickinson and Armstrong ${ }^{42}$ and Bujard et al. ${ }^{40}$ up to $2000 \mathrm{~K}$. Although the values of Bolef and de Klerk ${ }^{41}$ measured below $500 \mathrm{~K}$ and the data of Bujard et al. above $2000 \mathrm{~K}$ are noticeably higher than our $\gamma_{1}$ function, the values still overlap within their mutual uncertainties. Our $\gamma_{1}(T)$ function is also consistent with the low-pressure asymptotic values of McQueen et $a .^{3}$ and Al'tshuler et al., ${ }^{5}$ as discussed in Section V B.

The three black lines with different dashing styles in Figure 4 are $\gamma(T)$ values computed from the best fit functions $\gamma(V)$ listed in lines 2-4 of Table II. It appears that our final maximum likelihood $\gamma(V)$ function, obtained from the double-constrained fit, is virtually the same as the intermediate best-fit of selected Mo data to the Al'tshuler et al. $\gamma(V)$ model.

The noticeable discrepancy between our $\gamma_{1}(T)$ and $\gamma_{2}(T)$ values above $\sim 1000 \mathrm{~K}$, as discussed above, is most probably caused by melting of Mo along the hot Hugoniot, which would require estimates of $\alpha(T)$ and $C_{p}(T)$ for the liquid in order to correctly recalculate $\gamma_{2}(T)$ from $a(T)$ at high temperature. However, $\gamma_{2}(T)$ is shown at all temperatures for completeness and because it was found to be in good agreement with the data of Zeng et al. ${ }^{38}$ and consistent, within the uncertainties, with several other models. ${ }^{36,39,43,44}$

\section{Comparison with other EOS}

Inspection of Figure 4 indicates that all 1 bar macroscopic Grüneisen functions $\gamma(T)$ inferred from static highpressure, high-temperature data analysis $37,39,43,44$ or from first-principles calculations ${ }^{38}$ show only marginal agreement with the values recalculated from the experimental $K_{S}(T)$ data $^{40-42}$ or our EOS prediction $\gamma_{1}(T)$. None of the reported Mie-Grüneisen EOS functions for Mo constructed solely from static data can reproduce the low-pressure asymptotic values of $\gamma(T)$ as accurately as the EOS constructed from shock data only. The $\gamma(T)$ function reported by Guillermet and Grimvall ${ }^{37}$ looks like the best example. Yet, there is a significant discrepancy between their $\gamma(T)$ values and those we calculated using precisely the same $K_{s}(T)$ data from Bujard et al. ${ }^{40}$ The discrepancy is not due to the $C_{p}(T)$ functions; the specific heat we used is nearly identical to the relevant second derivative of the Gibbs energy function used by those authors. ${ }^{47}$ Rather, it appears that the discrepancy results from an oversimplified second order polynomial for $\alpha(T)$ used by the authors of Ref. 37. We tried all six post1985 accurate thermal expansion datasets available and average values from several combinations of these datasets and were unable to reproduce the negative slope of $\gamma(T)$ at 1 bar above $\sim 1800 \mathrm{~K}$ found in (and only in) the Grüneisen model of Ref. 37.

We now come (Figure 5) to those published $\gamma(T)$ functions ${ }^{44-46}$ that are plainly inconsistent with the experimental $\gamma(T)$ values from static measurements of $K_{S}(T)$ and with

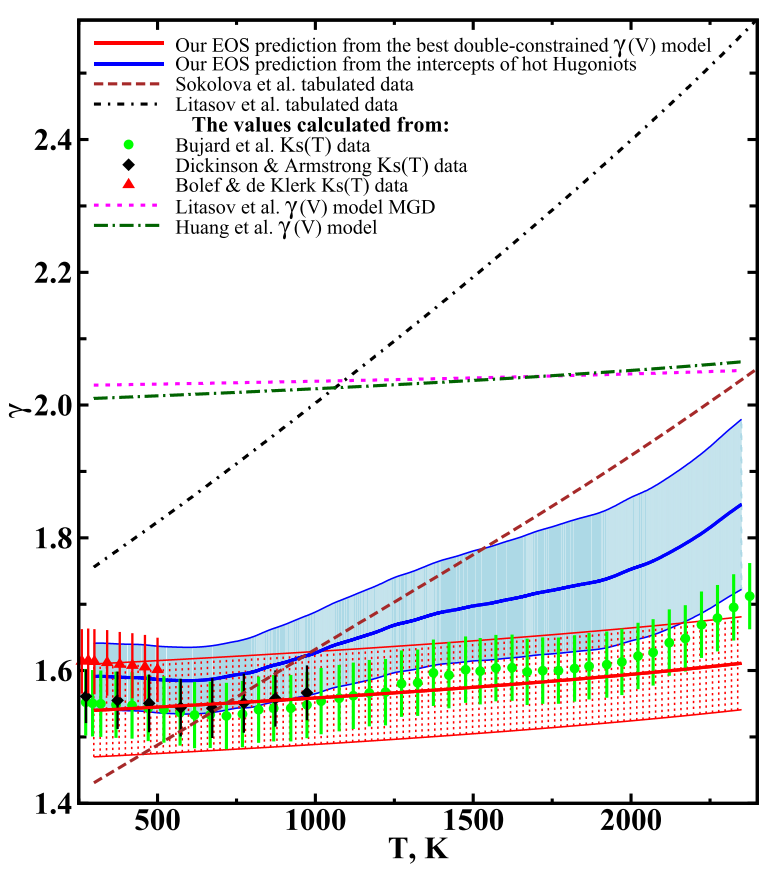

FIG. 5. Summary of macroscopic Grüneisen parameters at 1 bar $^{44-46}$ inconsistent with the results of our calculations from the reported measurements of $K_{s}(T)^{40-42}$ and the values predicted by our current EOS. Two shaded areas indicate the uncertainties of our $\gamma_{1}(T)$ (red) and $\gamma_{2}(T)$ (blue) data. Crosses at discrete data points are the estimated errorbars. Note the expanded scale compared to Fig. 4.

either the $\gamma_{1}(T)$ or $\gamma_{2}(T)$ functions predicted by our EOS fit to shock data. It is quite possible that each of these reported EOS functions ${ }^{44-46}$ still correctly predict thermal pressure or $P-V$ compression curves of molybdenum in the $P-T$ ranges intended. However, these EOS are unable to reproduce simultaneously the reported experimental elastic constants, thermal expansion, and specific heat data at elevated temperature.

\section{SUMMARY AND CONCLUSIONS}

An accurate family of Hugoniots for Mo shocked from various initial temperatures were constructed by review of all available data with stated or estimated uncertainties and application of state-of-the-art methods of general leastsquares fitting. We present Hugoniots for Mo with initial temperatures of 298, 1673, 1850, and $2300 \mathrm{~K}$ and parameterized functions for $\rho_{0}(T)$ and synthetic $D(T)$ vs. $U(T)$ lines valid from 298 to $2350 \mathrm{~K}$. All parameter uncertainties and essential correlation coefficients between these uncertainties were also computed and tabulated (see Section VI D of the supplementary material).

Our maximum likelihood solutions allow us to evaluate popular models for the volume dependence of the Grüneisen parameter and to constrain ambient Grüneisen coefficients for Mo with the highest accuracy permitted by the selected models and quality of available experimental data. Our choice of the simplest Grüneisen model, $\gamma=\gamma_{0} \times V / V_{0}$ with $\gamma_{0}=1.54 \pm 0.07$, was validated by (1) good agreement with the reported $\gamma_{0}$ values of 1.52 and 1.61 obtained, respectively, by McQueen et al. ${ }^{3}$ and Al'tshuler et al..$^{5}$ from their measurements on porous samples; (2) predicted values of 
bulk sound speed on the principal Hugoniot above the melting line in better agreement with the reported experimental data than similar predictions made with any other known Mo EOS; and (3) very good agreement between the $\gamma(T)$ values at 1 bar recalculated from our EOS $\gamma(V)$ model and those from the reported $K_{S}(T)$ data. Some noticeable deviation of our 1 bar $\gamma_{2}(T)$ values from the reported experimental data and our $\gamma_{1}(T)$ above, c.a., $1000 \mathrm{~K}$ is presumably caused by Mo melting. This does not imply a significant thermodynamic inconsistency of our EOS; it simply makes our prediction of macroscopic Grüneisen parameters from the Hugoniot intercepts only roughly valid at high temperatures.

It would be interesting to compare the results of our narrow-range EOS and the most advanced wide-range semiempirical $\mathrm{EOS}^{48}$ available for Mo. Unfortunately, the information reported in Ref. 48 was insufficient to compute the thermodynamic parameters of Mo required for a direct comparison with our model prediction.

\section{SUPPLEMENTARY MATERIAL}

See supplementary material for further computational details, relevant equations, and the tabular data from experiments and calculations.

\section{ACKNOWLEDGMENTS}

O.V.F. thanks Professor I. V. Lomonosov of IPCP RAS, Russia, for numerous stimulating discussions on the subject and Professor S. Brandt of Siegen University, Germany, for his clarification on the origin of the most accurate leastsquares fitting method reported. This work was supported by the U.S. NSF, Award Nos. EAR-0810116 and EAR1426526.

${ }^{1}$ O. V. Fat'yanov, P. D. Asimow, and T. J. Ahrens, AIP Conf. Proc. 1195, $855-858$ (2010).

${ }^{2}$ O. V. Fat'yanov and P. D. Asimow, J. Phys.: Conf. Ser. 500, 062003 (2014).

${ }^{3}$ R. G. McQueen, S. P. Marsh, J. W. Taylor, J. N. Fritz, and W. J. Carter, in High-Velocity Impact Phenomena, edited by R. Kinslow (Academic Press, New York, 1970), p. 542.

${ }^{4}$ S. P. Marsh, LASL Shock Hugoniot Data (University of California Press, Berkeley, 1980).

${ }^{5}$ L. V. Al'tshuler, S. E. Brusnikin, and E. A. Kuz'menkov, Z. Prikl. Mekh. Tekh. Fiz. 1, 134 (1987) [J. Appl. Mech. Tech. Phys. 28, 129 (1987)].

${ }^{6}$ R. S. Hixson and J. N. Fritz, J. Appl. Phys. 71, 1721 (1992).

${ }^{7}$ J. Wang, F. Coppari, R. F. Smith, J. H. Eggert, A. E. Lazicki, D. E. Fratanduono, J. R. Rygg, T. R. Boehly, G. W. Collins, and T. S. Duffy, Phys. Rev. B 92, 174114 (2015).

${ }^{8}$ R. S. Hixson, D. A. Boness, J. W. Shaner, and J. A. Moriarty, Phys. Rev. Lett. 62, 637 (1989).

${ }^{9}$ P. D. Asimow, D. Sun, and T. J. Ahrens, Phys. Earth Planet. Int. 174, 302 (2009).

${ }^{10}$ J. H. Nguyen, M. C. Akin, R. Chau, D. E. Fratanduono, W. P. Ambrose, O. V. Fatyanov, P. D. Asimow, and N. C. Holmes, Phys. Rev. B 89, 174109 (2014); 92, 026102 (2015).

${ }^{11}$ J. M. Walsh, M. H. Rice, R. G. McQueen, and F. L. Yarger, Phys. Rev. 108, 196 (1957).

${ }^{12}$ R. G. McQueen and S. P. Marsh, J. Appl. Phys. 31, 1253 (1960).

${ }^{13}$ K. K. Krupnikov, A. A. Bakanova, M. I. Brazhnik, and R. F. Trunin, Dokl. Akad. Nauk SSSR 148, 1302 (1963) [Sov. Phys. -Dokl. 8, 205 (1963)].

${ }^{14}$ A. C. Mitchell, W. J. Nellis, J. A. Moriarty, R. A. Heinle, N. C. Holmes, R. E. Tipton, and G. W. Repp, J. Appl. Phys. 69, 2981 (1991).
${ }^{15}$ M. D. Furnish and L. C. Chhabildas, in High Strain Rate Behavior of Refractory Metals and Alloys, edited by R. Asfahani, E. Chen, and A. Crowson (The Minerals, Metals, and Materials Society, Warrendale, PA, 1992), p. 229.

${ }^{16}$ L. V. Al'tshuler, A. A. Bakanova, I. P. Dudoladov, E. A. Dynin, R. F. Trunin, and B. S. Chekin, Z. Prikl. Mekh. Tekh. Fiz. 2, 3 (1981) [J. Appl. Mech. Tech. Phys. 22, 145 (1981)].

${ }^{17}$ R. F. Trunin, M. A. Podurets, G. V. Simakov, L. V. Popov, and A. G. Sevast'yanov, Teplofiz. Vys. Temp. 32, 786 (1994) [High Temp. 32, 734 (1994)].

${ }^{18}$ A. C. Mitchell and W. J. Nellis, J. Appl. Phys. 52, 3363 (1981).

${ }^{19}$ N. N. Kalitkin and L. V. Kuz'mina, in High-Pressure Shock Compression of Solids VII, edited by V. E. Fortov, L. V. Al'tshuler, R. F. Trunin, and A. I. Funtikov (Springer-Verlag, New York, 2004), p. 109.

${ }^{20} \mathrm{R}$. Böck, Application of a Generalized Method of Least Squares for Kinematical Analysis of Tracks of Bubble Chamber Photographs (CERN, Geneva, 1960), p. CERN 60-30.

${ }^{21}$ S. Brandt, in Statistical and Computational Methods in Data Analysis, 1st ed. (North-Holland, Amsterdam, 1970), p. 181; typos corrected in the 4th ed. (Springer International, Switzerland, 2014), p. 251.

${ }^{22} \mathrm{C}$. Heiles, "Least- and Chi-squares for the budding aficionado: Art and practice," June 12, 2002, see http://micro.ustc.edu.cn/IDL/lsfit_2002.pdf or http://www.sos.siena.edu/ jmoustakas/idl_tutorials/calidl_lsfit.pdf.

${ }^{23}$ G. H. Miller, T. J. Ahrens, and E. M. Stolper, J. Appl. Phys. 63, 4469 (1988).

${ }^{24}$ T. S. Duffy and T. J. Ahrens, J. Appl. Phys. 76, 835 (1994).

${ }^{25}$ S. Root, L. Shulenburger, R. W. Lemke, D. H. Dolan, T. R. Mattsson, and M. P. Desjarlais, Phys. Rev. Lett. 115, 198501 (2015).

${ }^{26}$ L. R. Fokin and V. Ya. Chekhovskoi, Teplofiz. Vys. Temp. 29, 94 (1991)[High Temp. 29, 89 (1991)].

${ }^{27}$ K. Wang and R. R. Reeber, Mater. Sci. Eng. R23, 101 (1998).

${ }^{28}$ V. Yu. Bodryakov, Teplofiz. Vys. Temp. 52, 863 (2014) [High Temp. 52, 840 (2014)]

${ }^{29}$ M. W. Chase, Jr., "NIST-JANAF thermochemical tables," J. Phys. Chem. Ref. Data, Monogr. 9, 1578-1580 (1998).

${ }^{30}$ P. D. Desai, J. Phys. Chem. Ref. Data 16, 91 (1987).

${ }^{31}$ R. G. McQueen, J. N. Fritz, and S. P. Marsh, J. Geophys. Res. 69, 2947, doi:10.1029/JZ069i014p02947 (1964); R. G. McQueen, S. P. Marsh, and J. N. Fritz, ibid. 72, 4999, doi:10.1029/JZ072i020p04999 (1967).

${ }^{32}$ A. M. Molodets, Fiz. Goreniya Vzryva 31, 132 (1995) [Combust. Explos. Shock Waves 31, 620 (1995)].

${ }^{33}$ A. M. Molodets, High Pressure Res. 25, 211 (2005).

${ }^{34}$ A. V. Bushman and V. E. Fortov, Sov. Phys. -Usp. 26, 465 (1983) [Usp. Fiz. Nauk 140, 177 (1983)].

${ }^{35}$ W. Liu, Q. Liu, M. L. Whitaker, Y. Zhao, and B. Li, J. Appl. Phys. 106, 043506 (2009).

${ }^{36}$ V. M. Elkin, V. N. Mikhailov, and T. Yu. Mikhailova, Fiz. Metall. Metall. 112, 563 (2011) [Phys. Metals Metallogr. 112, 535 (2011)].

${ }^{37}$ A. F. Guillermet and G. Grimvall, Phys. Rev. B 44, 4332 (1991).

${ }^{38}$ Z. Y. Zeng, C. E. Hu, L. C. Cai, X. R. Chen, and F. Q. Jing, J. Phys. Chem. B 114, 298 (2010).

${ }^{39}$ P. I. Dorogokupets, T. S. Sokolova, B. S. Danilov, and K. D. Litasov, Geodyn. Tektonophys. 3, 129 (2012); An online calculator of reported thermodynamic parameters is available at http://labpet.crust.irk.ru/ count.jsp?base_id=125\&fold_id=2\&fold_name $=\mathrm{T}-\mathrm{P}$ and http://labpet. crust.irk.ru/count.jsp?base_id $=126 \&$ fold_id $=3 \&$ fold_name $=T-X$.

${ }^{40}$ P. Bujard, R. Sanjines, E. Walker, J. Ashkenazi, and M. Peter, J. Phys. F: Metal Phys. 11, 775 (1981).

${ }^{41}$ D. I. Bolef and J. de Klerk, J. Appl. Phys. 33, 2311 (1962).

${ }^{42}$ J. M. Dickinson and P. E. Armstrong, J. Appl. Phys. 38, 602 (1967).

${ }^{43}$ L. Burakovsky and D. L. Preston, J. Phys. Chem. Solids 65, 1581 (2004).

${ }^{44}$ K. D. Litasov, P. I. Dorogokupets, E. Ohtani, Y. Fei, A. Shatskiy, I. S. Sharygin, P. N. Gavryushkin, S. V. Rashchenko, Yu. V. Seryotkin, Y. Higo, K. Funakoshi, A. D. Chanyshev, and S. S. Lobanov, J. Appl. Phys. 113, 093507 (2013).

${ }^{45}$ T. S. Sokolova, P. I. Dorogokupets, and K. D. Litasov, Russian Geol. Geophys. 54, 181 (2013).

${ }^{46}$ X. Huang, F. Li, Q. Zhou, Y. Meng, K. D. Litasov, X. Wang, B. Liu, and T. Cui, Sci. Rep. 6, 19923 (2016).

${ }^{47}$ A. F. Guillermet, Int. J. Thermophys. 6, 367 (1985).

${ }^{48}$ V. E. Fortov and I. V. Lomonosov, Phys. -Usp. 57, 219 (2014) [Usp. Fiz. Nauk 184, 231 (2014)] I. V. Lomonosov, AIP Conf. Proc. 1793, 050022 (2017). 